presented in Table II. Comparison of the frequencies listed in Table II with the Raman frequencies recently published by Rosenbaum, von Grosse and Jacobson ${ }^{4}$ shows a general correspondence in pattern in the range below 1200 $\mathrm{cm}^{-1}$. The degeneracies present in the frequencies of Table II would be removed by terms in the potential energy involving the interaction of adjacent bonds. For results of quantitative significance, such interactions should of course be included.

For very long molecules distribution functions of the Blackman type for the high and low frequency branches can be obtained from Eq. (10) and employed in the calculation of the vibrational partition function of the molecule. Thus the frequency densities $g_{+}(\nu)$ and $g_{-}(\nu)$ would be

$$
\begin{aligned}
& g_{+}(\nu)=\frac{1}{\bar{\nu}}\left(\frac{d f_{+}}{d l}\right)^{-1}, \\
& g_{-}(\nu)=\frac{1}{\bar{\nu}}\left(\frac{d f_{-}}{d l}\right)^{-1} .
\end{aligned}
$$

\footnotetext{
${ }^{4}$ Rosenbaum, v. Grosse and Jacobson, J. Am. Chem. Soc. 61, 689 (1939).
}

J U L Y , 1939

JOURNAL OF CHEMICAL PHYSICS

The distribution functions are rather complicated and are best presented graphically. This will be done at a later time.

From the present discussion, it appears that the infinite chain treatment of polyatomic molecules has distinct possibilities as a method for determining the normal modes of long-chain molecules. With the inclusion of higher order interactions and three-dimensional branching to provide for the hydrogen atoms, it should give a rather good approximation for molecules of moderate length. For short molecules, it can of course only serve as a method for the rough location of the frequencies. However, it seems possible that even here, if combined with perturbation theory, it might serve as the first step in a process of successive approximation. The frequencies which it gives are the exact frequencies of the finite segment under the influence of a known perturbation due to forces acting on its terminal atoms from the neighboring segments of the infinite chain.

In conclusion, the writer wishes to thank Professor G. B. Kistiakowsky for some interesting discussions which led to this investigation.

\title{
The Centrifugal Distortion of Axial Molecules
}

\author{
Z. I. Slawsky and David M. Dennison \\ University of Michigan, Ann Arbor, Michigan
}

(Received May 1, 1939)

The theory of the semi-rigid rotator developed by Wilson and Howard has been applied to the axial molecules $\mathrm{YX}_{3}$ and $Z Y X_{3}$. It is found that the change in rotational energy, $\delta W$, caused by the centrifugal distortion, can be expressed in terms of the quantum numbers $J$ and $K$ and as a function of the potential constants and the molecular dimensions. The formula is evaluated explicitly for $\mathrm{NH}_{3}$, and $\mathrm{ND}_{3}$, and for the former is, in $\mathrm{cm}^{-1}$ :

$$
\begin{aligned}
\frac{\delta W_{J K}}{h C}=-0.000625 J^{2}(J+1)^{2}+0.000950 K^{2} J(J+1) \\
+0.000799 J(J+1)-0.000630 K^{4}-0.00189 K^{2}
\end{aligned}
$$

The pure rotation lines of $\mathrm{NH}_{3}$ and of $\mathrm{ND}_{3}$ are calculated

$\mathrm{R}$ ECENTLY two interesting papers by Wilson and Howard ${ }^{1}$ and by Wilson ${ }^{2}$ have appeared

\footnotetext{
${ }^{1}$ E. B. Wilson and J. B. Howard, J. Chem. Phys. 4, 260 (1936).

${ }^{2}$ E. B. Wilson, J. Chem. Phys. 4, 313 (1936).
}

and compared with the observations of Wright and Randall, and of Barnes. The agreement is very satisfactory. The theory predicts that the rotation lines are multiple. The spacing of this fine structure was too small to be observable in the region mapped by Wright and Randall but should be possible to detect in the case of the higher members of the rotation series.

We have also calculated the pure rotation spectrum of $\mathrm{PH}_{3}$ by using Howard's approximate values of the dimensions and force constants of this molecule. The agreement with experimental values is as good as could be expected from such rough data.

which deal with the vibration rotation energy levels of polyatomic molecules. In these papers there is developed, along general lines, the effect of the centrifugal force stretching upon the 
rotational levels. The experimental existence of such an effect has been observed in the far infrared for $\mathrm{NH}_{3}$ and $\mathrm{PH}_{3}$ by Wright and Randall ${ }^{3}$ who found that the pure rotation lines of these molecules are not linearly spaced-as must be expected for a rigid rotator-but that their positions are given by a formula of the type: $\nu=A J-B J^{3}$. The constant $B$ is very small, indicating that the centrifugal force stretching, although clearly observable, is a higher order effect. Similar observations have been made by Barnes ${ }^{4}$ on $\mathrm{ND}_{3}$ and have been confirmed for the molecule $\mathrm{NH}_{3}$ by the measurements of Houston ${ }^{5}$ on the Raman spectrum of that molecule.

We propose to apply the theory developed by Wilson and Howard to tetratomic and pentatomic axial molecules and to obtain explicit expressions for the change in the rotational energy. We shall show that these expressions are in very good agreement with the available experimental data.

Howard and Wilson have derived a general expression for the Hamiltonian of a semi-rigid polyatomic molecule which is rather complicated. However, they show that if one is interested only in the rotational distortion of the nonvibrating molecule, the expression may be considerably simplified. Under these circumstances the energy, which is purely rotational and therefore suitable for calculating the pure rotation spectrum, takes the form,

$$
\begin{aligned}
W_{R}=\frac{1}{2} \sum_{\alpha \beta} \mu_{\alpha \beta}{ }^{(0)} & P_{\alpha} P_{\beta} \\
& -\frac{1}{8} \sum_{\alpha \beta \gamma \delta \delta} \frac{\mu_{\alpha \beta}{ }^{(k)} \mu_{\gamma \delta}{ }^{(k)}}{\lambda_{k}} P_{\alpha} P_{\beta} P_{\gamma} P_{\delta} .
\end{aligned}
$$

The indices $\alpha, \beta, \gamma, \delta$ represent the directions in a rotating system of rectangular coordinates $x, y$, $z$, which will be defined in the next paragraph. The $P$ 's are matrices giving the components of the total angular momentum of the molecule along the coordinate axes. The index $k$ denotes a normal mode of vibration and $\lambda_{k}$ is connected to the normal frequency of vibration in the usual way, $\lambda_{k}=4 \pi^{2} \nu_{k}^{2}$.

The $\mu_{\alpha \beta}{ }^{(k)}$ are determined by the moments and

\footnotetext{
${ }^{3}$ N. Wright and H. M. Randall, Phys. Rev. 44, 391 (1933).

${ }^{4}$ R. B. Barnes, Phys. Rev. 47, 658 (1935).

${ }^{5}$ W. V. Houston, Phys. Rev. 44, 903 (1933).
}

products of inertia. Consider a rotating coordinate system whose origin is fixed at the center of gravity of the molecule, and whose axes are defined by the conditions that the angular momentum of the molecule with respect to them shall be zero at all times. The moments and products of inertia $A, B, C, D, E$ and $F$ relative to these axes are now calculated. Since the molecule is only semi-rigid, they will evidently be functions of the mutual displacements of the particles as well as of the equilibrium distances. It is convenient to express them in terms of the normal coordinates which are designated by $Q_{1} \cdots Q_{n}$. A determinant is now built from the moments and products of inertia.

$$
\Delta=\left|\begin{array}{rrr}
A & -D & -F \\
-D & B & -E \\
-F & -E & C
\end{array}\right|
$$

$\mu_{\alpha \beta}$ is the $\alpha \beta$ cofactor of $\Delta$ divided by $\Delta$ itself. $\mu_{\alpha \beta}{ }^{(0)}$ is its equilibrium value, that is, $\mu_{\alpha \beta}$ in which the normal coordinates $Q_{1} \cdots Q_{n}$ have all been set equal to zero. Thus the first term of Eq. (1) is the familiar expression for the rotational energy of a rigid molecule. The first derivative of $\mu_{\alpha \beta}$ with respect to $Q_{k}$ evaluated at the equilibrium position is given the symbol $\mu_{\alpha \beta}{ }^{(k)}$.

From the dimensions of the molecule we shall calculate the $\mu_{\alpha \beta}{ }^{(k)}$ which are of course constants; then find a representation for the $P$ 's which satisfy the exchange rules but which in general will not make $W$ diagonal. The final step will be to diagonalize $W$ and thus obtain the eigenvalues of our problem.

\section{The Axial Molecule $\mathrm{YX}_{3}$}

Consider the molecule $\mathrm{YX}_{3}$. The geometrical configuration is such that the atoms occupy the corners of a regular pyramid, the $\mathrm{Y}$ atom at its vertex and the three $\mathrm{X}$ atoms at the corners of the equilateral triangular base. See Fig. 1.

The moments of inertia $A, B$ and $C$ and the products of inertia $D, E$ and $F$ of such molecules, may be readily calculated to the approximation in which only first powers in the displacements of the atoms from their equilibrium positions are retained. 


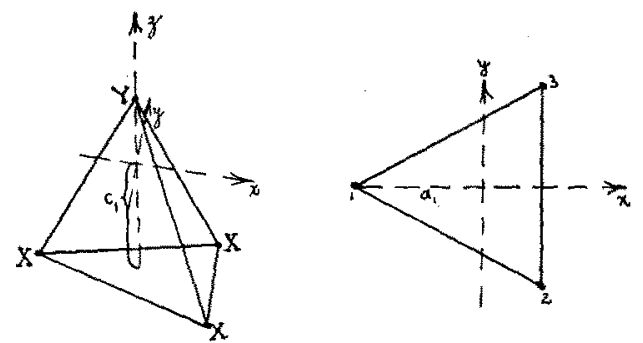

Fic. 1 .

$A=A_{0}-2 m c_{1}\left(z_{1}+z_{2}+z_{3}-3 z_{4}\right)-m a_{1} \sqrt{ } 3\left(y_{2}-y_{3}\right)$,

$B=B_{0}-2 m c_{1}\left(z_{1}+z_{2}+z_{3}-3 z_{4}\right)-m a_{1}\left(2 x_{1}-x_{2}-x_{3}\right)$,

$C=C_{0}-m a_{1} \sqrt{ } 3\left(y_{2}-y_{3}\right)-m a_{1}\left(2 x_{1}-x_{2}-x_{3}\right)$,

$D=-\frac{1}{2} m a\left(2 y_{1}-y_{2}-y_{3}\right)-\frac{\sqrt{ } 3}{2} m a_{1}\left(x_{2}-x_{3}\right)$,

$E=-m c_{1}\left(y_{1}+y_{2}+y_{3}-3 y_{4}\right)-\frac{\sqrt{3}}{2} m a_{1}\left(z_{2}-z_{3}\right),$.

$F=-m c_{1}\left(x_{1}+x_{2}+x_{3}-3 x_{4}\right)-\frac{1}{2} m a_{1}\left(2 z_{1}-z_{2}-z_{3}\right)$.

The coordinates $x_{1}, x_{2}, x_{3}$, and $x_{4}$ are the displacements from the equilibrium positions along the $x$ direction of the three $\mathrm{X}$ atoms and the $\mathrm{Y}$ atom, respectively; $y_{1}, y_{2}, y_{3}$, and $y_{4}$ are similar displacements in the $y$ direction and $z_{1}, z_{2}, z_{3}$, and $z_{4}$ along the $z$ direction. $A_{0}$ and $C_{0}$ are the equilibrium values of the moments of inertia. $A_{0}$ is the moment of inertia about an axis through the center of gravity of the molecule and perpendicular to the geometrical axis of symmetry. $C_{0}$ is the moment of inertia about this axis of symmetry. $c_{1}$ is the distance of the center of gravity of the molecule from the plane of the $\mathrm{X}$ atoms, $a_{1}$ is the distance of the $\mathrm{X}$ atoms from the symmetry axis. $m$ is the mass of an $\mathrm{X}$ atom.

These expressions may be considerably simplified when the symmetry of the molecule and the conservation of linear and angular momenta are taken into account. A coordinate system which does this will now be introduced. That one needs only six coordinates is evident from the fact that the $\mathrm{YX}_{3}$ molecule has twelve degrees of freedom, three of which belong to the translation of the molecule, and three to its rotation. Hence only six coordinates are necessary for the six internal degrees of freedom of the molecule. The six

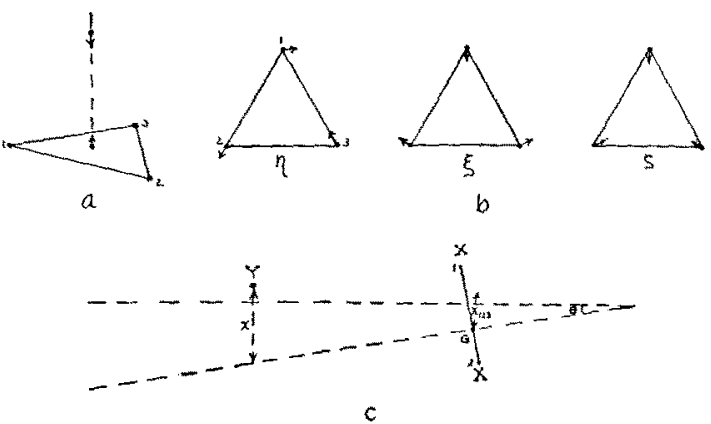

Fio. 2.

coordinates we shall use are illustrated in Fig. 2. The coordinate $z$ gives the displacement of the $\mathrm{Y}$ atom with respect to the center of gravity of the $\mathrm{X}$ atoms, which takes place along the geometrical axis of symmetry. The coordinates $x$ or $y$ measure the displacement of the $\mathrm{Y}$ atom from a line drawn perpendicular to the plane of the $\mathrm{X}$ atoms and through their center of gravity. The angle $\theta$, which describes the tipping of the base with respect to the $Y$ atom, is determined by the conservation of angular momentum, say about an axis through the center of gravity of the $\mathrm{X}$ atoms and perpendicular to the plane of the paper in Fig. 2c. The coordinates $\xi, \eta$ and $\zeta$ are indicated in Fig. 2b. They are so chosen that the actual displacements are given by $\xi / \sqrt{ } 3$, $\eta / \sqrt{ } 3$ and $\xi / \sqrt{ } 3$. Four of these coordinates $x, y$, $\xi, \eta$ are the same as those used by Johnston and Dennison ${ }^{6}$ and are discussed in their paper.

In terms of the six coordinates thus introduced, $A, B, C, D, E$ and $F$ assume a simpler form.

$$
\begin{aligned}
& A=A_{0}+6 m c_{1} z+\sqrt{ } 3 m a_{1}(\zeta+\xi), \\
& B=B_{0}+6 m c_{1} z+\sqrt{ } 3 m a_{1}(\zeta-\xi), \\
& C=C_{0}+2 \sqrt{ } 3 m a_{1} \zeta, \\
& D=\sqrt{ } 3 m a_{1} \eta, \\
& E=6 m c_{1}\left(C_{0} / 2 A_{0}\right) y, \\
& F=6 m c_{1}\left(C_{0} / 2 A_{0}\right) x .
\end{aligned}
$$

To introduce normal coordinates it is necessary to set up the usual determinant of the coefficients in the potential and kinetic energies of the molecule.

${ }^{6}$ M. Johnston and D. M. Dennison, Phys. Rev. 48, 868 (1935). 
It is well known that axially symmetrical molecules such as $\mathrm{YX}_{3}$, possess four normal frequencies which are separated into two symmetry classes, two parallel and two perpendicular frequencies. Since these belong to different symmetry classes, the Hamiltonian representing the vibrational motion will fall into two parts with no interaction terms. Thus one really has two distinct problems. The parallel frequencies are given by the coordinates $z$ and $\zeta$ while the motions corresponding to the perpendicular frequencies are given by $x, y, \xi$ and $\eta$. The kinetic and potential energies are then

$$
\begin{aligned}
& 2 T_{\mathrm{II}}=\mu \dot{z}^{2}+m \dot{\zeta}^{2}, \\
& 2 V_{\mathrm{H}}=a z^{2}+b \xi^{2}+2 c z \zeta, \\
& 2 T_{\perp}=m \kappa\left(\dot{x}^{2}+\dot{y}^{2}\right)+m\left(\dot{\xi}^{2}+\dot{\eta}\right), \\
& 2 V_{\perp}=\alpha\left(x^{2}+y^{2}\right)+\beta\left(\xi^{2}+\eta^{2}\right)+2 \gamma(x \xi+y \eta),
\end{aligned}
$$

where

$$
\mu=\frac{3 m M}{3 m+M} \text { and } \quad \kappa=\frac{C_{0}}{2 A_{0}} \frac{\mu}{m} ;
$$

$a, b, c, \alpha, \beta$ and $\gamma$ are the potential constants.

The determinants which, when set equal to zero, yield the normal frequencies are

$$
\left|\begin{array}{cc}
\mu \lambda-a & -c \\
-c & m \lambda-b
\end{array}\right|=0, \quad\left|\begin{array}{cc}
m \kappa \lambda-\alpha & -\gamma \\
-\gamma & m \lambda-\beta
\end{array}\right|=0 .
$$

The first gives the parallel frequencies and the second, the doubly degenerate perpendicular frequencies. The relations between $x, y, z, \xi, \eta, \zeta$ and the normal coordinates $Q_{1}, Q_{2}, Q_{3}, Q_{4}, Q_{6}$ and $Q_{8}$ follow immediately,

$$
\begin{array}{ll}
x=A_{2} Q_{2}+A_{4} Q_{4}, & \xi=B_{2} Q_{2}+B_{4} Q_{4}, \\
y=A_{6} Q_{6}+A_{8} Q_{8}, & \eta=B_{6} Q_{6}+B_{8} Q_{8}, \\
z=A_{1} Q_{1}+A_{3} Q_{3}, & \zeta=B_{1} Q_{1}+B_{3} Q_{3} .
\end{array}
$$

The subscripts are so chosen that the odd ones correspond to the parallel frequencies while the even subscripts belong to the perpendicular frequencies. The coefficients in these transformation equations are the normalized cofactors of the determinants.

$$
A_{i}=\frac{m \lambda_{i}-\beta}{\left(m \kappa\left(m \lambda_{i}-\beta\right)^{2}+m \gamma^{2}\right)^{\frac{1}{2}}},
$$

$$
\begin{array}{r}
B_{i}=\frac{\gamma}{\left(m \kappa\left(m \lambda_{i}-\beta\right)^{2}+m \gamma^{2}\right)^{\frac{1}{2}}}, \\
i=2,4,6,8, \\
A_{j}=\frac{m \lambda_{j}-b}{\left(\mu\left(m \lambda_{j}-b\right)^{2}+m c^{2}\right)^{\frac{1}{2}}}, \\
B_{j}=\frac{c}{\left(\mu\left(m \lambda_{j}-b\right)^{2}+m c^{2}\right)^{\frac{1}{2}}}, \\
j=1,3 .
\end{array}
$$

Inasmuch as the motions of the atoms in the plane perpendicular to the axis of symmetry are degenerate, $A_{2}=A_{6}, A_{4}=A_{8}, B_{2}=B_{6}$ and $B_{4}=B_{8}$.

The moments and products of inertia $A, B, C$, $D, E$ and $F$ may now be written in terms of the normal coordinates and the determinant $\Delta$ set up. Since all powers of the displacements above the first are rejected in our calculations, the determinant and all its cofactors may be readily expanded and the $\mu_{\alpha \beta}{ }^{(k)}$ evaluated. If the coefficients of the matrices $P_{\alpha} P_{\beta} P_{\gamma} P_{\delta}$ are designated by $\tau_{\alpha \beta \gamma \delta}$, that is,

$$
\tau_{\alpha \beta \gamma \delta} \equiv \sum_{k} \frac{\mu_{\alpha \beta}{ }^{(k)} \mu_{\gamma \delta}(k)}{\lambda_{k}},
$$

their values are

$$
\begin{aligned}
\tau_{x x x x}= & \frac{9 m^{2} a_{1}{ }^{2}}{A_{0}{ }^{4} \lambda_{1}}\left(\frac{2 c_{1}}{a_{1}} A_{1}+\frac{B_{1}}{\sqrt{ }}\right)^{2} \\
& +\frac{9 m^{2} a_{1}{ }^{2}}{A_{0}{ }^{4} \lambda_{3}}\left(\frac{2 c_{1}}{a_{1}} A_{3}+\frac{B_{3}}{\sqrt{ } 3}\right)^{2} \\
& \quad+\frac{9 m^{2} a_{1}{ }^{2}}{3 A_{0}{ }^{4} \lambda_{2}} B_{2}{ }^{2}+\frac{9 m^{2} a_{1}{ }^{2}}{3 A_{0}{ }^{4} \lambda_{4}} B_{4}{ }^{2}, \\
\tau_{y y y y}= & \tau_{x x x x}, \quad \\
\tau_{z z z z}= & \frac{12 m^{2} a_{1}{ }^{2}}{C_{0}{ }^{4} \lambda_{1}} B_{1}{ }^{2}+\frac{12 m^{2} a_{1}{ }^{2}}{C_{0}{ }^{4} \lambda_{3}} B_{3}{ }^{2}, \\
\tau_{x x y y}= & \frac{9 m^{2} a_{1}{ }^{2}}{A_{0}{ }^{4} \lambda_{1}}\left(\frac{2 c_{1}}{a_{1}} A_{1}+\frac{B_{1}}{\sqrt{3}}\right)^{2} \\
& +\frac{9 m^{2} a_{1}{ }^{2}}{A_{0}{ }^{4} \lambda_{3}}\left(\frac{2 c_{1}}{a_{1}} A_{3}+\frac{B_{3}}{\sqrt{3}}\right)^{2} \\
& -\frac{9 m^{2} a_{1}{ }^{2}}{3 A_{0}{ }^{4} \lambda_{2}} B_{2}{ }^{2}-\frac{9 m^{2} a_{1}{ }^{2}}{3 A_{0}{ }^{4} \lambda_{4}} B_{4}{ }^{2},
\end{aligned}
$$


note that $\tau_{x x y y}=\tau_{x x x x}-2 \tau_{x y x y}$,

$$
\begin{aligned}
\tau_{x x z z}= & \frac{18 m^{2} a_{1}^{2}}{\sqrt{ } 3 C_{0}{ }^{2} A_{0}{ }^{2} \lambda_{1}} B_{1}\left(\frac{2 c_{1}}{a_{1}} A_{1}+\frac{B_{1}}{\sqrt{ } 3}\right) \\
& +\frac{18 m^{2} a_{1}^{2}}{\sqrt{ } 3 C_{0}{ }^{2} A_{0}{ }^{2} \lambda_{3}} B_{3}\left(\frac{2 c_{1}}{a_{1}} A_{3}+\frac{B_{3}}{\sqrt{ } 3}\right),
\end{aligned}
$$

$\tau_{y y z z}=\tau_{x x z z}$,

$$
\begin{aligned}
\tau_{x y x y} & =\frac{9 m^{2} a_{1}{ }^{2}}{3 A_{0}{ }^{4} \lambda_{2}} B_{2}{ }^{2}+\frac{9 m^{2} a_{1}{ }^{2}}{3 A_{0}{ }^{4} \lambda_{4}} B_{4}{ }^{2}, \\
\tau_{y z y z} & =\frac{36 m^{2} c_{1}{ }^{2}}{C_{0}{ }^{2} A_{0}{ }^{2} \lambda_{2}} A_{2}{ }^{2}+\frac{36 m^{2} c_{1}{ }^{2}}{C_{0}{ }^{2} A_{0}{ }^{2} \lambda_{4}} A_{4}{ }^{2}, \\
\tau_{z x z x} & =\tau_{y z y z}, \\
\tau_{x y y z} & =\frac{9 m^{2} a_{1} c_{1}}{\sqrt{ } 3 A_{0}{ }^{4} \lambda_{2}} A_{2} B_{2}+\frac{9 m^{2} a_{1} c_{1}}{\sqrt{ } 3 A_{0}{ }^{4} \lambda_{4}} A_{4} B_{4}, \\
\tau_{x x z x} & =-\tau_{x y y z}, \\
\tau_{y y z x} & =\tau_{x y y z} .
\end{aligned}
$$

The above expressions constitute all the coefficients which are different from zero. The perturbation which we are calculating is given by considering all the possible permutations of $\alpha, \beta$, $\gamma$ and $\delta$ in such terms as $\tau_{\alpha \beta \gamma \delta} P_{\alpha} P_{\beta} P_{\gamma} P_{\delta}$. The $P_{\alpha} P_{\beta} P_{\gamma} P_{\delta}$ may be collected into six groups, each of which has the same coefficient. Thus :

$$
\begin{aligned}
& \tau_{x x x x}\left\{P_{x} P_{x} P_{x} P_{x}+P_{y} P_{y} P_{y} P_{y}+P_{x} P_{x} P_{y} P_{y}\right. \\
& \tau_{z z z z} P_{z} P_{z} P_{z} P_{z}, \\
& \left.+P_{y} P_{y} P_{x} P_{x}\right\} \\
& \tau_{x x z z}\left\{P_{x} P_{x} P_{z} P_{z}+P_{z} P_{z} P_{x} P_{x}+P_{y} P_{y} P_{z} P_{z}\right. \\
& \left.+P_{z} P_{z} P_{y} P_{y}\right\} \\
& \tau_{x y x y}\left\{P_{x} P_{y} P_{x} P_{y}+P_{y} P_{x} P_{y} P_{x}+P_{x} P_{y} P_{y} P_{x}\right. \\
& \left.+P_{y} P_{x} P_{x} P_{y}-2 P_{x} P_{x} P_{y} P_{y}-2 P_{y} P_{y} P_{x} P_{x}\right\} \text {, } \\
& \tau_{y z y z}\left\{P_{y} P_{z} P_{y} P_{z}+P_{z} P_{y} P_{z} P_{y}+P_{y} P_{z} P_{z} P_{y}\right. \\
& +P_{z} P_{y} P_{y} P_{z}+P_{z} P_{z} P_{z} P_{z}+P_{z} P_{z} P_{z} P_{z} \\
& \left.+P_{x} P_{z} P_{z} P_{x}+P_{z} P_{x} P_{x} P_{z}\right\} \text {, } \\
& \tau_{x y y z}\left\{P_{x} P_{y} P_{y} P_{z}+P_{x} P_{y} P_{z} P_{y}+P_{y} P_{x} P_{y} P_{z}\right. \\
& +P_{y} P_{x} P_{z} P_{y}+P_{y} P_{z} P_{x} P_{y}+P_{y} P_{z} P_{y} P_{x} \\
& +P_{z} P_{y} P_{x} P_{y}+P_{z} P_{y} P_{y} P_{x}+P_{y} P_{y} P_{x} P_{z} \\
& +P_{y} P_{y} P_{z} P_{x}+P_{z} P_{x} P_{y} P_{y}+P_{x} P_{z} P_{y} P_{y} \\
& -P_{x} P_{x} P_{z} P_{x}-P_{x} P_{x} P_{x} P_{z}-P_{x} P_{z} P_{x} P_{x} \\
& \left.-P_{z} P_{x} P_{x} P_{x}\right\} \text {. }
\end{aligned}
$$

All the other $P_{\alpha} P_{\beta} P_{\gamma} P_{\delta}$ 's have vanishing coefficients. Now by means of the well-known commutation rules for such matrices,

$$
P^{2}=P_{x}^{2}+P_{y}^{2}+P_{z}^{2},
$$

$P^{2}$ commutes with all three

$$
\begin{aligned}
& P_{x} P_{y}-P_{y} P_{x}=(\hbar / i) P_{z}, \\
& P_{y} P_{z}-P_{z} P_{y}=(\hbar / i) P_{x}, \\
& P_{z} P_{x}-P_{x} P_{z}=(\hbar / i) P_{y},
\end{aligned}
$$

the above may be reduced to much simpler matrices :

$$
\begin{aligned}
& \tau_{x x x x}\left(P^{4}-2 P^{2} P_{z}^{2}+P_{z}^{4}\right), \\
& \tau_{z z z z} P_{z}^{4}, \\
& \tau_{x x z z}\left(2 P^{2} P_{z}^{2}-2 P_{z}^{4}\right), \\
& \tau_{x y x y}\left(5 \hbar^{2} P_{z}^{2}-2 \hbar^{2} P^{2}\right), \\
& \tau_{y z y z}\left(4 P^{2} P_{z}^{2}-5 \hbar^{2} P_{z}^{2}-4 P_{z}^{4}+\hbar^{2} P^{2}\right), \\
& \tau_{x y y z}\left(8 P_{y}^{2} P_{z} P_{x}+8 P_{x} P_{z} P_{y}{ }^{2}-2 P^{2} P_{x} P_{z}-2 P^{2} P_{z} P_{x}\right. \\
& \left.\quad-4 P_{z}^{2} P_{x}+6 P_{z}{ }^{2} P_{x} P_{z}+6 P_{z} P_{x} P^{2}-4 P_{z} P_{z}^{2}\right) .
\end{aligned}
$$

The eigenvalues of these matrices can now be calculated. There are many matrix representations which will satisfy the four matrix equations arising from the commutation rules. One such representation has the following nonvanishing elements :

$$
\begin{aligned}
& \left(P_{x}\right)_{J, K+1}^{J, K}=i\left(P_{y}\right)_{J, K+1}^{J, K}=\frac{\hbar}{2}((J-K)(J+K+1))^{\frac{1}{3}}, \\
& \left(P_{z}\right)_{J K}^{J K}=\hbar K, \quad J \geqslant K .
\end{aligned}
$$

This representation is chosen because in it the angular momentum $P_{z}$ and the square of the total angular momentum $P^{2}$ become diagonal matrices. In this representation the $W$ matrix has the following nonvanishing elements (in waves per $\mathrm{cm}$ ):

$$
\begin{gathered}
\frac{W_{J K} J K}{h c_{L}}=\frac{\hbar^{2}}{2 A_{0} h c_{L}} J(J+1)+\left(\frac{1}{C_{0}}-\frac{1}{A_{0}}\right) \frac{\hbar^{2}}{h c_{L}} K^{2} \\
-P_{1} J^{2}(J+1)^{2}+2\left(P_{1}-P_{3}-2 P_{5}\right) K^{2} J(J+1) \\
+\left(2 P_{4}-P_{5}\right) J(J+1)-\left(P_{1}+P_{2}-2 P_{3}-4 P_{5}\right) K^{4} \\
-5\left(P_{4}-P_{5}\right) K^{2}
\end{gathered}
$$


and the off diagonal elements

$$
\frac{W_{J, K \pm 1}^{J K}}{h c_{L}}, \frac{W_{J, K \pm 3}^{J K}}{h c_{L}}
$$

which are of the same order of magnitude as the last five terms in the expression above. $h$ is Planck's constant, $c_{L}$, the velocity of light and the $P$ 's are defined by

$$
\begin{gathered}
P_{1}=\frac{\tau_{x x x x} \hbar^{4}}{8 h c_{L}}, \quad P_{2}=\frac{\tau_{z z z z} \hbar^{4}}{8 h c_{L}}, \quad P_{3}=\frac{\tau_{x x z z} \hbar^{4}}{8 h c_{L}}, \\
P_{4}=\frac{\tau_{x y x y} \hbar^{4}}{8 h c_{L}}, \quad P_{5}=\frac{\tau_{y z y z} \hbar^{4}}{8 h c_{L}} .
\end{gathered}
$$

It may be shown that to a first-order approximation the off diagonal elements can be neglected. To this approximation, therefore, the $W$ matrix is diagonal with elements given by expression (2).

Thus far the P's have been given in terms of the normalized first minors of the determinants formed by the coefficients of the kinetic and potential energies. One may show, however, that:

$$
\begin{gathered}
\frac{A_{1}{ }^{2}}{\lambda_{1}}+\frac{A_{3}{ }^{2}}{\lambda_{3}}=\frac{b}{a b-c^{2}}, \quad \frac{A_{2}{ }^{2}}{\lambda_{2}}+\frac{A_{4}{ }^{2}}{\lambda_{4}}=\frac{\beta}{\alpha \beta-\gamma^{2}}, \\
\frac{B_{1}{ }^{2}}{\lambda_{1}}+\frac{B_{3}{ }^{2}}{\lambda_{3}}=\frac{a}{a b-c^{2}}, \quad \frac{B_{2}{ }^{2}}{\lambda_{2}}+\frac{B_{4}{ }^{2}}{\lambda_{4}}=\frac{\alpha}{\alpha \beta-\gamma^{2}}, \\
\frac{A_{1} B_{1}}{\lambda_{1}}+\frac{A_{3} B_{3}}{\lambda_{3}}=-\frac{c}{a b-c^{2}}, \\
\frac{A_{2} B_{2}}{\lambda_{2}}+\frac{A_{4} B_{4}}{\lambda_{4}}=-\frac{\gamma}{\alpha \beta-\gamma^{2}},
\end{gathered}
$$

so that the $P$ 's have the more easily calculable form:

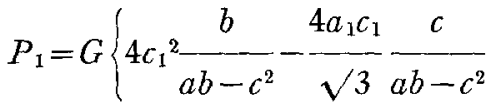

$$
\begin{aligned}
& \left.+\frac{a_{1}^{2}}{3} \frac{a}{a b-c^{2}}+\frac{a_{1}^{2}}{3 \cdot} \frac{\alpha}{\alpha \beta-\gamma^{2}}\right\},
\end{aligned}
$$

$$
\begin{aligned}
& P_{2}=\frac{1}{4}\left(\frac{2 A_{0}}{C_{0}}\right)^{4} G\left\{\frac{\left.a_{1}^{2}-\frac{a}{3 a b-c^{2}}\right\},}{P_{3}}=\frac{1}{2}\left(\frac{2 A_{0}}{C_{0}}\right)^{2} G\left\{-\frac{2 a_{1} c_{1}}{\sqrt{ } 3 a b-c^{2}}+\frac{c}{3} \frac{a_{1}^{2}}{a b-c^{2}}\right\},\right.
\end{aligned}
$$$$
P_{4}=G\left\{\frac{a_{1}{ }^{2}}{3 \alpha \beta-\gamma^{2}}\right\},
$$$$
P_{5}=G\left\{c_{1}^{2} \frac{\beta}{\alpha \beta-\gamma^{2}}\right\} \text {, }
$$

where $G \equiv 9 m^{2} \hbar^{4} / 8 c_{L} h A_{0}{ }^{4}$.

Expression (2) is perfectly general insofar as it will hold for any ammonia-like molecule as for example $\mathrm{ND}_{3}, \mathrm{PH}_{3}, \mathrm{PD}_{3}, \mathrm{AsH}_{3}, \mathrm{AsD}_{3}$, etc. The numerical calculation involves the dimensions of the particular molecule and its force constants. These are of ten obtainable from the vibrational spectrum, and the fine structure of these vibrational bands. The dimensions of both $\mathrm{NH}_{3}$, and $\mathrm{ND}_{3}$ are well known from the work of Migeotte and Barker, ${ }^{7}$ Wright and Randall, ${ }^{3}$ Dennison and Uhlenbeck. ${ }^{8}$ The force constants for these two molecules have also been evaluated by Migeotte and Barker but in coordinates which are different from ours. The transformation to our coordinates is, however, straight forward.

The various constants for $\mathrm{NH}_{3}$ are:

$$
\begin{aligned}
a & =5.46 \times 10^{5} \text { dynes } / \mathrm{cm}, \\
b & =5.04 \times 10^{5} \text { dynes } / \mathrm{cm}, \\
c & =4.23 \times 10^{5} \text { dynes } / \mathrm{cm}, \\
A_{0} & =2.782 \times 10^{-40} \mathrm{~g} \mathrm{~cm}{ }^{2}, \\
a_{1} & =0.950 \times 10^{-8} \mathrm{~cm}, \\
\alpha & =10.16 \times 10^{5} \text { dynes } / \mathrm{cm}, \\
\beta & =3.54 \times 10^{5} \text { dynes } / \mathrm{cm}, \\
\gamma & =3.67 \times 10^{5} \text { dynes } / \mathrm{cm}, \\
C_{0} & =4.497 \times 10^{-40} \mathrm{~g} \mathrm{~cm}, \\
c_{1} & =0.296 \times 10^{-8} \mathrm{~cm} .
\end{aligned}
$$

The constants for $\mathrm{ND}_{3}$ are identical with those for $\mathrm{NH}_{3}$ except that $A_{0}=5.397 \times 10^{-40}, C_{0}=8.985$ $\times 10^{-40}$ and $c_{1}=0.275 \times 10^{-8}$. These values give for $\delta W$, the change in the energy due to the

${ }^{7}$ M. V. Migeotte and E. F. Barker, Phys. Rev. 50, 418 (1936).

${ }^{8}$ D. M. Dennison and G. E. Uhlenbeck, Phys. Rev. 41, 313 (1932). 
rotational distortion :

$$
\begin{aligned}
\frac{\delta W}{h c_{L}}= & -0.000625 J^{2}(J+1)^{2} \\
& +0.000950 K^{2} J(J+1) \\
& +0.000799 J(J+1) \\
& \quad-0.000630 K^{4}-0.00189 K^{2}
\end{aligned}
$$

and for $\mathrm{ND}_{3}$,

$$
\begin{aligned}
\frac{\delta W}{h c_{L}}= & -0.000176 J^{2}(J+1)^{2} \\
& +0.000261 K^{2} J(J+1)+0.000226 J(J+1) \\
& -0.000163 K^{4}-0.000540 K^{2} .
\end{aligned}
$$

The pure rotation frequencies are then obtained by means of the selection rules. For all ammonia-like molecules these are: $\Delta K=0$ and $\Delta J= \pm 1$. The frequencies are then given by:

$$
\begin{aligned}
\nu_{J}^{J-1}=\frac{\hbar^{2}}{A_{0} h c_{L}} J+ & 0.00160 J \\
& -0.00250 J^{3}+0.00190 K^{2} J
\end{aligned}
$$

for $\mathrm{NH}_{3}$ and

$$
\begin{aligned}
\nu_{J}^{J-1}=\frac{\hbar^{2}}{A_{N} h c_{L}} J & +0.000452 J \\
& -0.000704 J^{3}+0.000522 K^{2} J
\end{aligned}
$$

for $\mathrm{ND}_{3}$.

It is evident from the last term in (5) and (6) that the far infra-red lines of these molecules have a fine structure. For $\mathrm{NH}_{3}$ the spacing is given by $0.00190 J(2 K-1)$ where $K$ is taken for the larger component. This spacing is much too small to be observed for lines with $J$ values less than 15. However, their presence will affect the position of the peak of any experimentally observed line. Such a peak will be roughly at the center of gravity of the fine structure components. Therefore, in order to compare the theoretical values with those observed, one must consider the intensity pattern of a given line and determine its center of gravity.

The rotation lines of $\mathrm{NH}_{3}$ consist of close doublets which are caused by the existence of a double minimum for the $\mathrm{N}$ atom. This complica- tion must be considered in calculating the intensity patterns.

The double minimum problem has been treated by several authors. ${ }^{8,9}$ It is shown that in the pure-rotation spectrum there are two vibrational energy levels for each value of $J$, one of these belongs to the symmetric solutions the other to the antisymmetric. The first are commonly designated by $\alpha$ and the latter by $\beta$. The selection rules are such that only transitions from $\alpha$ to $\beta$ or $\beta$ to $\alpha$ are allowed. The doublet member on the long wave-length side corresponds to the $\beta$ to $\alpha$ transition while the other arises from the $\alpha$ to $\beta$ transition. The doublet separation for the case of pure rotation will be just twice the separation of the $\alpha$ and $\beta$ levels in the zeroth vibrational state and is independent of $J$.

The intensity formula has been given explicitly by Dennison and Hardy ${ }^{9}$ and may be immediately adapted to our case.

$$
I=A \nu_{K^{\prime}}{ }^{K}[x] \frac{J^{2}-K^{2}}{J} e^{-\sigma\left(J^{2}+J\right)-\sigma \beta K^{2}}\left(1-e^{-h \nu / K T}\right) .
$$

Since only the shape of the line, rather than its absolute intensity is of interest, we may reject all multiplicative constants, thus:

$$
I \cong \nu_{K}, K[x]\left(J^{2}-K^{2}\right) e^{-\sigma \beta K^{2}}\left(1-e^{-h_{\nu} / K T}\right),
$$

$\sigma \equiv \hbar^{2} / 2 A_{0} K T$ and $\beta=\left(A_{0} / C_{0}-1\right) .[x]$ is the weight factor which has the following values: if the component under consideration is due to a transition for which $K=0, J$ even, and the final state $\alpha$, then $[x]=0$; if the final state is $\beta$, then $[x]=2$. If $J$ is odd, then the converse is true. For $K \neq 0$ and not a multiple of 3 , then regardless of whether the final state is an $\alpha$ or a $\beta$ state, $[x]=1$. If $K$ is a multiple of 3 , then $[x]=2$ for both $\alpha$ and $\beta$ final states.

As an example, the results of the calculation will be given in detail for $J=7$. In Table I the (-) sign designates the long wave-length component of the doublet. The exact position of the maximum when such lines are unresolved is, of course, difficult to predict. It was assumed that it is given by the center of gravity of the components. We have, therefore, $\Delta_{+}=0.187$ and

9 Dennison and Hardy, Phys. Rev. 39, 938 (1932). Dennison, Rev. Mod. Phys. 3, 208 (1931). F. Hund, Zeits. f. Physik 43, 805 (1927). 
$\Delta_{-}=0.144$ where the $\Delta^{\prime}$ 's are measured from the component for which $K=0$, and hence from the position where the maximum would be if there were no $J K^{2}$ term. The experimental curve for this line as observed by Wright and Randall together with the theoretically calculated components are shown in Fig. 3. For $J=6, \Delta_{+}=0.074$ and $\Delta_{-}=0.103$ and for $J=5$, the third line observed by Wright and Randall, $\Delta_{+}=0.064$ and $\Delta_{-}=0.042$.

The exact frequencies may now be calculated and compared with the observed frequencies. Since the doublet separation is independent of $J$, we take the average value, i.e., $1.32 \mathrm{~cm}^{-1}$. The single adjustable parameter, the $A_{0}$ moment of inertia, may be adjusted to fit any one of the frequencies and the rest then calculated. With $\hbar^{2} / A_{0} h c_{L}=19.890$ we have the results shown in Table II.

This comparison together with Fig. 3 which shows that the observed curve fits the calculated components well, indicates that the theory is adequate for the explanation of this spectrum.

Barnes ${ }^{4}$ has mapped the far infra-red spectrum of $\mathrm{NH}_{3}$ for $J$ values up to 12 , but unfortunately the resolving power is not as good as that obtained by Wright and Randall and the results, therefore, not quite so accurate. The doublet separation is not in evidence. We have calculated a few of these lines to see how well our calculations fit the observed lines. In doing so, the intensity patterns of the two members of the doublet were superimposed, and the center of gravity of the resultant pattern taken as the position of the peak of the line. (Table III.)

The pure rotation spectrum of $\mathrm{ND}_{3}$ is completely determined by the data already known from $\mathrm{NH}_{3}$. The dimensions and the force constants are the same for both molecules and the

TABLE I.

\begin{tabular}{c|c|c|c|c|c|c}
\hline & & \multicolumn{2}{|c|}{$\left(J^{2}-K^{2}\right)[x]$} & & & \\
\cline { 3 - 5 }$K$ & \multirow{2}{*}{$0.0019 J K^{2}$} & $(-)$ & $(+)$ & B.F. & $I_{-}$ & \multicolumn{1}{|c|}{$I_{+}$} \\
\hline 0 & 0.000 & 98.0 & 0.0 & 1.000 & 98.0 & 0.0 \\
1 & 0.013 & 48.0 & 48.0 & 1.019 & 48.9 & 48.9 \\
2 & 0.053 & 45.0 & 45.0 & 1.076 & 48.4 & 48.4 \\
3 & 0.122 & 80.0 & 80.0 & 1.180 & 94.4 & 94.4 \\
4 & 0.214 & 33.0 & 33.0 & 1.343 & 44.4 & 44.4 \\
5 & 0.324 & 24.0 & 24.0 & 1.587 & 38.0 & 38.0 \\
6 & 0.480 & 26.0 & 26.0 & 1.947 & 50.6 & 50.6 \\
\hline
\end{tabular}

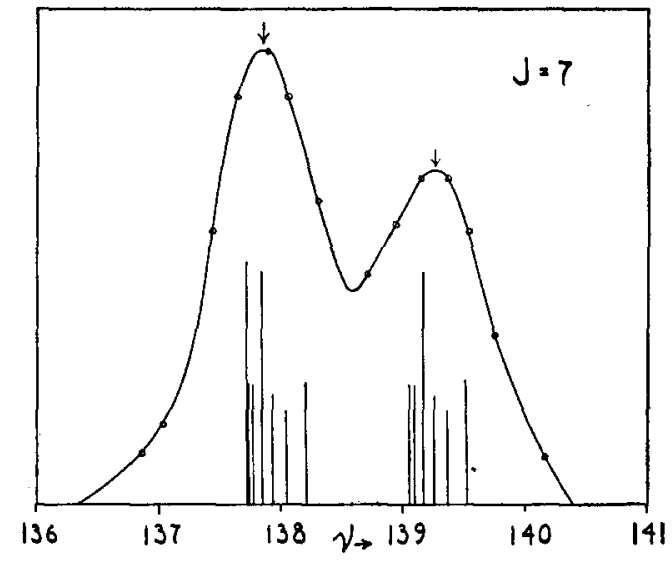

FIG. 3 .

moments of inertia may be calculated from the mass of deuterium and the dimensions of the molecule. The frequencies are given by:

$$
\begin{aligned}
& \nu_{J}{ }^{J-1}=10.260 J+0.000452 J \\
&-0.000704 J^{3}+0.000522 K^{2} J .
\end{aligned}
$$

Barnes has mapped the $\mathrm{ND}_{3}$ lines with the same arrangement he used for $\mathrm{NH}_{3}$ and presumably with the same accuracy. In Table IV some of the calculated lines are compared with the experimental values.

It is perhaps of interest to note that the usual procedure of fitting a cubic expression such as $A J-B J^{3}$ to the observed frequencies gives very nearly the correct values. From theoretical considerations, however, a $J K^{2}$ term should be included. It turns out that the center of gravity of the components is roughly given by one-third the coefficient of the $J K^{2}$ term multiplied by $J^{3}$ so that the theoretical values will approximately follow a simple cubic expression. The divergence from this simple formula will, of course, become evident when $J$ values are high enough to spread out the fine structure components.

We have also calculated the far infra-red spectrum of $\mathrm{PH}_{3}$, four lines of which have been mapped by Wright and Randall under high resolving power. These calculations suffer, however, from the fact that the infra-red frequencies of this molecule are not well known so that neither the dimensions nor the force constants can be determined with any degree of accuracy. We have taken the values as given by Howard. ${ }^{10}$

${ }^{10}$ J. B. Howard, J. Chem. Phys. 3, 207 (1935). 
He calculates the force constants on the assumption that the molecule is of the valence type. Under this assumption only two fundamental frequencies are needed to determine the two force constants, which he calls $K$ and $H . K$ is the constant which gives the resistance to the stretching of the $\mathrm{P}-\mathrm{H}$ bond and $\mathrm{H}$ is the constant which gives the resistance to the deformation of the $\mathrm{H}-\mathrm{P}-\mathrm{H}$ angle. The values are: $K=3.09 \times 10^{5}$ and $H=0.34 \times 10^{5}$ dynes $/ \mathrm{cm}$. The dimensions of this molecule may be estimated and give for the moment of inertia, $A_{0}, 6.221$ $\times 10^{-40}$, and for $C_{0}, 7.73 \times 10^{-40}$. The dimension, $a_{1}$, is $1.24 \times 10^{-8}$ and $c_{1}$ is $0.657 \times 10^{-8}$. With these values one may deduce the general force constants, $a, b, c$, and $\alpha, \beta, \gamma$, from the constants $\mathrm{K}$ and $\mathrm{H}$. They turn out to be:

$$
\begin{aligned}
a & =3.63 \times 10^{5} \text { dynes } / \mathrm{cm}, \\
b & =7.39 \times 10^{5} \text { dynes } / \mathrm{cm}, \\
c & =3.26 \times 10^{5} \text { dynes } / \mathrm{cm}, \\
\alpha & =3.97 \times 10^{5} \text { dynes } / \mathrm{cm}, \\
\beta & =1.61 \times 10^{5} \text { dynes } / \mathrm{cm}, \\
\gamma & =1.53 \times 10^{5} \text { dynes } / \mathrm{cm} .
\end{aligned}
$$

The pure rotation energy of $\mathrm{PH}_{3}$ is then given by:

$$
\begin{aligned}
& \frac{W_{J K}^{J K}}{h c_{L}}=\frac{\hbar^{2}}{2 A_{0} h c_{L}} J(J+1)+\left(\frac{1}{C_{0}}-\frac{1}{A_{0}}\right) \frac{\hbar^{2} K^{2}}{h c_{L}} \\
& -0.000123 J^{2}(J+1)^{2}+0.000155 K^{2} J(J+1) \\
& \quad+0.000104 J(J+1)-0.000105 K^{4}-0.000208 K^{2} .
\end{aligned}
$$

Since the selection rules are identically the same as those for $\mathrm{NH}_{3}$, the change in the energy due to the centrifugal distortion is, in waves per $\mathrm{cm}$ :

$$
\Delta \nu_{J}{ }^{J-1}=0.000208 J+0.000310 J K^{2}-0.000492 J^{3} \text {. }
$$

It must be pointed out that neither the fundamental frequencies nor the dimensions are really well established for $\mathrm{PH}_{3}$. The above formulae are therefore somewhat inaccurate.

TABLE II.

\begin{tabular}{c|r|r|r}
\hline \hline$J$ FINAL & \multicolumn{1}{|c|}{ CALC. } & \multicolumn{1}{c|}{ OHS. } & \multicolumn{1}{c}{ DEv. } \\
\hline 5 & 98.52 & 98.53 & +0.01 \\
5 & 99.86 & 99.83 & -0.03 \\
6 & 118.25 & 118.22 & -0.03 \\
6 & 119.54 & 119.58 & +0.04 \\
7 & 137.86 & 137.88 & +0.02 \\
7 & 139.23 & 139.21 & -0.02 \\
\hline \hline
\end{tabular}

They may, nevertheless, serve well for locating other pure rotation lines of phosphine. We have calculated one of the lines which have been observed by Wright and Randall $(J=13)$ and obtained 114.66 as against the observed value, 114.84 ; a fair check considering the inaccuracy of the data used.

\section{The $Z_{3}$ Molecule}

There are a number of molecules of the $Z_{Y X}$ type whose infra-red spectrum has been the subject of considerable study. Since all such molecules have a permanent electric dipole moment, they have a far infra-red spectrum. We propose to extend our calculations to such molecules and in particular evaluate numerically the spectrum of the methyl halides.

The geometrical configuration of the $Z_{3 X}$ molecule is indicated in Fig. 4. The $\mathrm{YX}_{3}$ group of atoms in this molecule has the same geometrical configuration as the $\mathrm{YX}_{3}$ molecule discussed in the previous section. The $Z$ atom is located along the axis of the $\mathrm{YX}_{3}$ pyramid.

In a manner similar to that used for the $\mathrm{YX}_{3}$ molecule, we may set up the moments of inertia, $A, B, C$, and the products of inertia, $D, E$, and $F$, in terms of the equilibrium positions of the atoms and of their displacements from equilibrium. The internal coordinates are then introduced by means of the conditions for the conservation of linear and angular momenta. For the conservation of linear momentum:

$$
\begin{aligned}
& M_{2} \dot{z}_{5}+M_{1} \dot{z}_{4}+3 m z_{123}=0, \\
& M_{2} \dot{x}_{5}+M_{1} \dot{x}_{4}+3 m \dot{x}_{123}=0, \\
& M_{2} \dot{y}_{5}+M_{1} \dot{y}_{4}+3 m \dot{y}_{123}=0,
\end{aligned}
$$

and for the conservation of the angular momentum:

$$
\begin{array}{cc}
M_{2} a_{0} \dot{x}_{5}+M_{1} b_{0} \dot{x}_{4}+\frac{1}{2} C_{0} \dot{\theta}=0, & a_{0} \equiv c_{3}+c_{1}, \\
M_{2} a_{0} \dot{y}_{5}+M_{1} b_{0} \dot{y}_{4}+\frac{1}{2} C_{0} \dot{\theta}=0, & b_{0}=c_{1}-c_{2} . \\
\text { TABLE III. } &
\end{array}
$$

\begin{tabular}{c|c|c|c}
\hline \hline$J$ FiNAL & CALC. & OBS. & DEV. \\
\hline 8 & 158.11 & 158.33 & -0.22 \\
9 & 177.58 & 177.69 & -0.11 \\
10 & 197.06 & 196.93 & +0.13 \\
11 & 216.32 & 216.61 & -0.29 \\
\hline
\end{tabular}


TABLE IV.

\begin{tabular}{c|c|c|c}
\hline$J$ FinAL & CALC. & OBS. & Dev. \\
\hline 10 & 102.08 & 102.32 & -0.24 \\
15 & 152.36 & 152.02 & +0.34 \\
16 & 162.13 & 162.12 & +0.01 \\
17 & 172.01 & 172.10 & -0.09 \\
18 & 181.88 & 181.99 & -0.11 \\
19 & 191.79 & 192.80 & -1.01 \\
20 & 201.59 & 202.10 & -0.51 \\
\hline
\end{tabular}

In the above equations $M_{2}, M_{1}$ and $m$ are the masses of the $Z, Y$ and the $\mathrm{X}$ atoms, respectively; $c_{1}$ is the equilibrium distance of the center of gravity of the three $\mathrm{X}$ atoms from the center of gravity of the molecule; $c_{2}$ and $c_{3}$ are the equilibrium distances of $Y$ and $Z$ atoms, respectively, from the center of gravity of the molecule; $x_{5}, y_{5}, z_{5}$ are the displacements of the $Z$ atom from equilibrium, $x_{4}, y_{4}, z_{4}$ that of the $\mathrm{Y}$ atom and $x_{123}, y_{123}, z_{123}$ are the displacements of any one of the $\mathrm{X}$ atoms from its position of equilibrium. $\theta$ is the angle between the perpendicular to the plane of the $\mathrm{X}$ atoms and the line drawn through the center of gravity of the $\mathrm{X}$ atoms, the $\mathrm{Y}$ and the $Z$ atoms. (See Fig. 5.) $C_{0}$ is, as in the case of $\mathrm{NH}_{3}$, the moment of inertia about the axis of symmetry when the molecule is considered rigid.

The coordinates which we introduce give the displacement of $Z$ with respect to $Y$ and $Y$ with respect to the center of gravity of the $\mathrm{X}$ atoms. Together with coordinates $\xi, \eta$ and $\zeta$, already described, we have the nine coordinates necessary to describe the internal motions of the molecule. The new coordinates are determined by:

$$
\begin{aligned}
& p=x_{5}-x_{4}, \quad q=y_{5}-y_{4}, \quad r=z_{5}-z_{4}, \\
& x=x_{4}-x_{123}, \quad y=y_{4}-y_{123}, \quad z=z_{4}-z_{123},
\end{aligned}
$$

thus

$$
\begin{aligned}
x_{123} & =-\frac{M_{2}}{\sum} p-\frac{M_{1}+M_{2}}{\sum} x, \\
x_{4} & =-\frac{M_{2}}{\sum} p+\frac{3 m}{\sum} x, \\
x_{5} & =+\frac{\sum^{\prime}}{\sum} p+\frac{3 m}{\sum} x,
\end{aligned}
$$

with similar expressions for $Y$ and $Z$.

$$
\theta=-\frac{2}{C_{0}}\left\{M_{2} c_{3} p+3 m c_{1} x\right\},
$$

here $\Sigma=M_{2}+M_{1}+3 m$ and $\Sigma^{\prime}=M_{1}+3 m$.

The moments and products of inertia expressed in these coordinates assume the following simple form:

$$
\begin{aligned}
& A=A_{0}+6 m c_{1} z+2 M_{2} c_{3} r+3 m a_{1}(\zeta+\xi), \\
& B=B_{0}+6 m c_{1} z+2 M_{2} c_{3} r+3 m a_{1}(\zeta-\xi), \\
& C=C_{0}+6 m a_{1} \zeta, \\
& D=3 m a_{1} \eta \\
& E=6 m c_{1} y+2 M_{2} c_{3} q, \\
& F=6 m c_{1} x+2 M_{2} c_{3} p .
\end{aligned}
$$

The coordinates $p$ and $x$ or $q$ and $y$ suffer the disadvantage of depending on the masses rather than on the geometric properties of the molecule. We therefore introduce the coordinates $X_{1}, X_{2}$ and $Y_{1}, Y_{2}$ as illustrated in Fig. 5.

$$
\begin{array}{ll}
X_{1}=p-\frac{a_{0}-b_{0}}{b_{0}} x, & Y_{1}=q-\frac{a_{0}-b_{0}}{b_{0}} y, \\
X_{2}=x-b_{0} \theta, & Y_{2}=y-b_{0} \theta .
\end{array}
$$

When these relations are solved for $p, x, q$ and $y$ and the values substituted in $E$ and $F$, they become:

$$
\begin{aligned}
& E=\frac{C_{0}}{2 A_{0}}\left\{2 M_{2} C_{3} Y_{1}+\frac{2}{b_{0}}\left(A_{0}-\frac{1}{2} C_{0}\right) Y_{2}\right\}, \\
& F=\frac{C_{0}}{2 A_{0}}\left\{2 M_{2} C_{3} X_{1}+\frac{2}{b_{0}}\left(A_{0}-\frac{1}{2} C_{0}\right) X_{2}\right\} .
\end{aligned}
$$

The normal coordinates are introduced by means
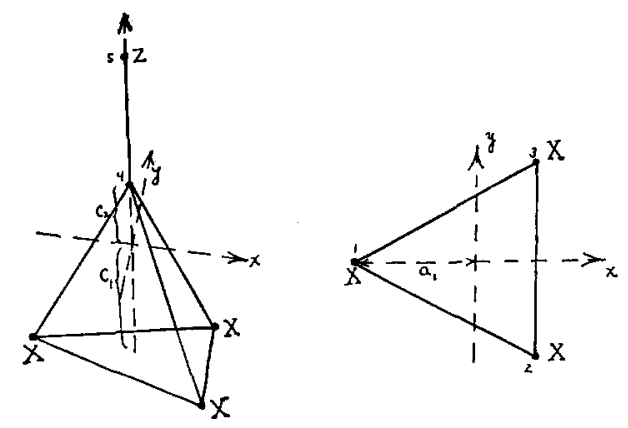

Fig. 4. 
of the usual determinant of the kinetic and potential energies. Just as in the case of $\mathrm{NH}_{3}$ the Hamiltonian for the $Z_{Y X}$ molecule splits up into two parts, one giving the motions belonging to the parallel bands and the other those giving the perpendicular bands.

The kinetic energy expressions for the parallel vibrations are:

$$
2 T_{11}=M_{2} \dot{z}_{5}^{2}+M_{1} \dot{z}_{4}^{2}+3 m \dot{z}_{123}^{2}+3 m \dot{\zeta}^{2},
$$

and for the perpendicular:

$$
2 T_{\perp}=M_{2} \dot{x}_{5}{ }^{2}+M_{1} \dot{x}_{4}{ }^{2}+3 m \dot{x}_{123}{ }^{2}+\frac{C_{0}}{2} \dot{\theta}^{2}+3 m \dot{\xi}^{2} .
$$

Since the expressions for the $y$ direction are always identical with those given above for the $x$ direction, we shall consistently omit them where no ambiguity exists.

Together with the expressions for the potential energies these may be written in terms of the coordinates described above.

$$
\begin{aligned}
& 2 T_{\mathrm{II}}=a_{11} \dot{r}^{2}+a_{33} \dot{z}^{2}+2 a_{13} \dot{r} \dot{z}+3 m \dot{\zeta}^{2}, \\
& 2 V_{\mathrm{II}}=a r^{2}+b z^{2}+c \zeta^{2}+2 d r z+2 e r \zeta+2 f z \zeta,
\end{aligned}
$$

and for the perpendiculars:

$$
\begin{aligned}
& 2 T_{\perp}=a_{22} \dot{X}_{1}^{2}+a_{44} \dot{X}_{2}{ }^{2}+2 a_{24} \dot{X}_{1} \dot{X}_{2}+3 m \dot{\xi}^{2}, \\
& 2 V_{\perp}=\alpha X_{1}{ }^{2}+\beta X_{2}{ }^{2}+\gamma \xi^{2}+2 \delta X_{1} X_{2}+2 \epsilon X_{1} \xi+2 \varphi X_{2} \xi .
\end{aligned}
$$

The constants $a, b, c, d, e, f, \alpha, \beta, \gamma, \delta, \epsilon$ and $\varphi$ are the usual force constants of the molecule. The evaluation of these constants is the subject of another paper. ${ }^{\text {" }}$ The other constants are as follows :

$$
\begin{aligned}
& a_{11}=\frac{M_{2} \Sigma^{\prime}}{\Sigma}, \quad a_{22}=\frac{3 m M_{1} M_{2} b_{0}{ }^{2}+M_{2}\left(C_{0} / 2\right) \Sigma^{\prime}}{A_{0} \Sigma}, \\
& a_{33}=\frac{3 m\left(M_{1}+M_{2}\right)}{\Sigma}, \quad a_{44}=\frac{\left(A_{0}-C_{0} / 2\right) C_{0} / 2}{A_{0} b_{0}^{2}}, \\
& a_{13}=\frac{3 m M_{2}}{\Sigma}, \quad a_{24}=\frac{M_{2}\left(3 m a_{0}+M_{1} a_{0}-M_{1} b_{0}\right) C_{0} / 2}{A_{0} b_{0} \Sigma} .
\end{aligned}
$$

Once the kinetic and potential energies are known, the transformation to the normal co-

\footnotetext{
11 Succeeding paper by the authors.
}

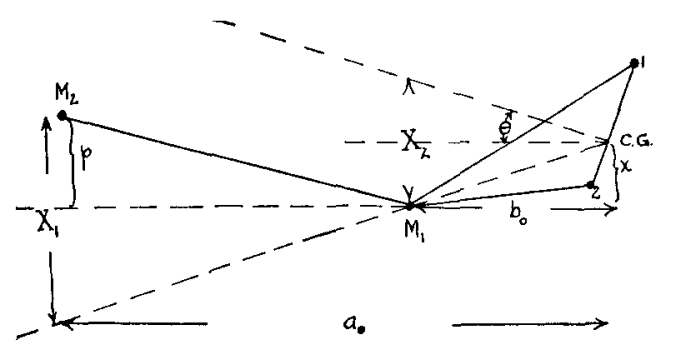

FIG. 5.

ordinates is given by:

$$
\begin{gathered}
z=A_{1} Q_{1}+A_{3} Q_{3}+A_{5} Q_{5}, \\
X_{2}=A_{2} Q_{2}+A_{4} Q_{4}+A_{6} Q_{6}, \\
\zeta=B_{1} Q_{1}+B_{3} Q_{3}+B_{5} Q_{5}, \\
\xi=B_{2} Q_{2}+B_{4} Q_{4}+B_{6} Q_{6}, \\
r=D_{1} Q_{1}+D_{3} Q_{3}+D_{5} Q_{5}, \\
X_{1}=D_{2} Q_{2}+D_{4} Q_{4}+D_{6} Q_{6} .
\end{gathered}
$$

The coefficients for $Y_{1}, Y_{2}$ and $\eta$ are identical with those for $X_{1}, X_{2}$ and $\xi$.

The coefficients $A_{1} \cdots D_{6}$ are the normalized first minors of the following determinants.

For the parallel frequencies,

$$
\left|\begin{array}{ccc}
a_{11} \lambda-a & a_{13} \lambda-d & -e \\
a_{13} \lambda-d & a_{33} \lambda-b & -f \\
-e & -f & 3 m \lambda-c
\end{array}\right|=0
$$

and for the perpendiculars:

$$
\left|\begin{array}{ccc}
a_{22} \lambda-\alpha & a_{24} \lambda-\delta & -\epsilon \\
a_{24} \lambda-\delta & a_{44} \lambda-\beta & -\varphi \\
-\epsilon & -\varphi & 3 m \lambda-\gamma
\end{array}\right|=0
$$

The first minors of these determinants are:

$$
\begin{aligned}
& U_{i}=\left(a_{33} e-a_{13} f\right) \lambda_{i}+d f-b e, \quad i=1,3,5, \\
& V_{i}=-\left\{\left(a_{13} e-a_{11} f\right) \lambda_{i}+a f-d e\right\}, \\
& W_{i}=\left(a_{11} a_{33}-a_{13}{ }^{2}\right) \lambda_{i}{ }^{2} \\
& -\left(a_{11} b+a_{33} a-2 a_{13} d\right) \lambda_{i}+a b-d^{2}, \\
& U_{j}=\left(a_{44} \epsilon-a_{24} \varphi\right) \lambda_{j}+\delta \varphi-\beta \epsilon, \quad j=2,4,6 \text {, } \\
& V_{j}=-\left\{\left(a_{24} \epsilon-a_{22} \varphi\right) \lambda_{j}+\alpha \varphi-\delta \epsilon\right\}, \\
& W_{j}=\left(a_{22} a_{44}-a_{24}{ }^{2}\right) \lambda_{j}{ }^{2}
\end{aligned}
$$$$
-\left(a_{22} \beta+a_{44} \alpha-2 a_{24} \delta\right) \lambda_{j}+\alpha \beta-\delta^{2} .
$$ 
The normalized first minors are then:

$$
\begin{aligned}
& D_{i}=\frac{U_{i}}{\left(a_{11} U_{i}^{2}+a_{33} V_{i}^{2}+2 a_{13} U_{i} V_{i}+3 m W_{i}^{2}\right)^{\frac{1}{2}}}, \\
& D_{i}=\frac{U_{j}}{\left(a_{22} U_{j}^{2}+a_{44} V_{j}^{2}+2 a_{24} U_{j} V_{j}+3 m W_{j}^{2}\right)^{\frac{1}{2}}}
\end{aligned}
$$

$A_{i}=\frac{V_{i}}{U_{i}} D_{i}, \quad B_{i}=\frac{W_{i}}{U_{i}} D_{i}, \quad A_{j}=\frac{V_{i}}{U_{j}} D_{j}, \quad B_{j}=\frac{W_{i}}{U_{j}} D_{j}$

Comparison with the $\mathrm{NH}_{3}$ case shows immediately that the matrices for this type of molecule fall into the same groupings. The coefficients are, of course, different and more complicated for numerical evaluation. For the same reasons as have been given for the $\mathrm{YX}_{3}$ molecule, the energy matrix, including the rotational distortion is diagonal to the order of approximation used here. The rotational energy levels are then given by an expression which formally is identical with (2). The $P$ 's, however, are now given by:

$$
\begin{aligned}
P_{1}=G & \left\{4 c_{1}{ }^{2}\left(\frac{A_{1}{ }^{2}}{\lambda_{1}}+\frac{A_{3}{ }^{2}}{\lambda_{3}}+\frac{A_{5}{ }^{2}}{\lambda_{5}}\right)\right. \\
& +4 a_{1} c_{1}\left(\frac{A_{1} B_{1}}{\lambda_{1}}+\frac{A_{3} B_{3}}{\lambda_{3}}+\frac{A_{5} B_{5}}{\lambda_{5}}\right) \\
& +a_{1}{ }^{2}\left(\frac{B_{1}{ }^{2}}{\lambda_{1}}+\frac{B_{3}{ }^{2}}{\lambda_{3}}+\frac{B_{5}{ }^{2}}{\lambda_{5}}\right) \\
& +\frac{4 M_{2}{ }^{2} c_{3}{ }^{2}}{9 m^{2}}\left(\frac{D_{1}{ }^{2}}{\lambda_{1}}+\frac{D_{3}{ }^{2}}{\lambda_{3}}+\frac{D_{5}{ }^{2}}{\lambda_{5}}\right) \\
& +\frac{24 m_{1} M_{2} c_{3}}{9 m^{2}}\left(\frac{A_{1} D_{1}}{\lambda_{1}}+\frac{A_{3} D_{3}}{\lambda_{3}}+\frac{A_{5} D_{5}}{\lambda_{5}}\right) \\
& +\frac{4 M_{2} c_{3} a_{1}}{3 m}\left(\frac{B_{1} D_{1}}{\lambda_{1}}+\frac{B_{3} D_{3}}{\lambda_{3}}+\frac{B_{5} D_{5}}{\lambda_{5}}\right) \\
P_{2}=\frac{1}{4}\left(\frac{2 A_{0}}{C_{0}}\right)^{4} G a_{1}{ }^{2}\left(\frac{B_{1}{ }^{2}}{\lambda_{1}}+\frac{B_{3}{ }^{2}}{\lambda_{3}}+\frac{B_{5}{ }^{2}}{\lambda_{5}}\right), & \left.+a_{1}{ }^{2}\left(\frac{B_{2}{ }^{2}}{\lambda_{2}}+\frac{B_{4}{ }^{2}}{\lambda_{4}}+\frac{B_{6}{ }^{2}}{\lambda_{6}}\right)\right\},
\end{aligned}
$$

$$
\begin{aligned}
P_{3}=\frac{1}{2}\left(\frac{2 A_{0}}{C_{0}}\right)^{2} G\left\{2 a_{1} c_{1}\left(\frac{A_{1} B_{1}}{\lambda_{1}}+\frac{A_{3} B_{3}}{\lambda_{3}}+\frac{A_{5} B_{5}}{\lambda_{5}}\right)\right. \\
+a_{1}^{2}\left(\frac{B_{1}^{2}}{\lambda_{1}}+\frac{B_{3}^{2}}{\lambda_{3}}+\frac{B_{5}{ }^{2}}{\lambda_{5}}\right) \\
\left.+\frac{2 M_{2} a_{1} C_{3}}{3 m}\left(\frac{B_{1} D_{1}}{\lambda_{1}}+\frac{B_{3} D_{3}}{\lambda_{3}}+\frac{B_{5} D_{5}}{\lambda_{5}}\right)\right\} \\
P_{4}=G a_{1}^{2}\left(\frac{B_{2}^{2}}{\lambda_{2}}+\frac{B_{4}^{2}}{\lambda_{4}}+\frac{B_{6}{ }^{2}}{\lambda_{6}}\right)
\end{aligned}
$$

$$
\begin{aligned}
P_{5}= & \left\{\frac{M_{2}^{2} c_{3}^{2}}{9 m^{2}}\left(\frac{D_{2}^{2}}{\lambda_{2}}+\frac{D_{4}{ }^{2}}{\lambda_{4}}+\frac{D_{6}{ }^{2}}{\lambda_{6}}\right)\right. \\
& +\frac{\left(A_{0}-C_{0} / 2\right)^{2}}{9 m^{2} b_{0}{ }^{2}}\left(\frac{A_{2}{ }^{2}}{\lambda_{2}}+\frac{A_{4}{ }^{2}}{\lambda_{4}}+\frac{A_{6}{ }^{2}}{\lambda_{6}}\right) \\
& \left.+\frac{2 M_{2} c_{3}}{9 m^{2} b_{0}}\left(A_{0}-\frac{C_{0}}{2}\right)\left(\frac{A_{2} D_{2}}{\lambda_{2}}+\frac{A_{4} D_{4}}{\lambda_{4}}+\frac{A_{6} D_{6}}{\lambda_{6}}\right)\right\}
\end{aligned}
$$

The numerical calculation of the $P$ 's turns out to be rather involved since it seems to be impossible to reduce them to expressions as simple as those for ammonia-like molecules. By using the dimensions and force constants discussed in the succeeding paper we obtained the values given in Table $\mathrm{V}$.

The change in the rotational energy levels in these methyl halides due to the centrifugal force stretching may then be immediately written as

$$
\begin{aligned}
\frac{W_{J K} J K}{h c_{L}}=\frac{\hbar^{2}}{2 A_{0} h c_{L}} J(J+1)+\left(\frac{1}{C_{0}}-\frac{1}{A_{0}}\right) \frac{\hbar^{2}}{h c_{L}} K^{2} \\
-4.34 \times 10^{-7} J^{2}(J+1)^{2} \\
+1.45 \times 10^{-5} K^{2} J(J+1) \\
-3.23 \times 10^{-6} J(J+1) \\
\quad-7.02 \times 10^{-4} K^{4}+1.67 \times 10^{-5} K^{2}
\end{aligned}
$$

for $\mathrm{FCH}_{3}$,

$$
\begin{aligned}
\frac{W_{J K^{J K}}}{h c_{L}}= & \frac{\hbar^{2}}{2 A_{0} h c_{L}} J(J+1)+\left(\frac{1}{C_{0}}-\frac{1}{A_{0}}\right) \frac{\hbar^{2}}{h c_{L}} K^{2} \\
& -1.14 \times 10^{-7} J^{2}(J+1)^{2} \\
& +0.976 \times 10^{-5} K^{2} J(J+1) \\
& -1.71 \times 10^{-6} J(J+1)-7.52 \times 10^{-4} K^{4} \\
& +0.861 \times 10^{-5} K^{2}
\end{aligned}
$$


TABLE V.

\begin{tabular}{rr|r|r|r|r}
\hline \hline & & \multicolumn{1}{|c|}{$\mathrm{FCH}_{3}$} & $\mathrm{ClCH}_{3}$ & \multicolumn{1}{|c|}{$\mathrm{BrCH}_{3}$} & \multicolumn{1}{|c}{$\mathrm{ICH}_{3}$} \\
\hline $10^{7}$ & $P_{1}$ & 4.34 & 1.14 & 0.30 & 0.38 \\
$10^{4}$ & $P_{2}$ & 6.88 & 7.43 & 8.46 & 10.83 \\
$10^{6}$ & $P_{3}$ & -13.70 & -8.23 & -4.71 & -6.18 \\
$10^{8}$ & $P_{4}$ & 10.75 & 1.04 & 0.22 & 0.096 \\
$10^{7}$ & $P_{5}$ & 34.46 & 17.32 & 6.94 & 5.30 \\
\hline \hline
\end{tabular}

for $\mathrm{ClCH}_{3}$,

$$
\begin{aligned}
\frac{W_{J K} J K}{h c_{L}}=\frac{\hbar^{2}}{2 A_{0} h c_{L}} J(J+1)+\left(\frac{1}{C_{0}}-\frac{1}{A_{0}}\right) \frac{\hbar^{2}}{h c_{L}} K^{2} \\
-0.30 \times 10^{-7} J^{2}(J+1)^{2} \\
+0.670 \times 10^{-5} K^{2} J(J+1) \\
-0.690 \times 10^{-6} J(J+1) \\
\quad-8.52 \times 10^{-4} K^{4}+0.346 \times 10^{-5} K^{2}
\end{aligned}
$$

for $\mathrm{BrCH}_{3}$, and

$$
\begin{aligned}
\frac{W_{J K}^{J K}}{h c_{L}}=\frac{\hbar^{2}}{2 A_{0} h c_{L}} J(J+1)+\left(\frac{1}{C_{0}}-\frac{1}{A_{0}}\right) \frac{\hbar^{2}}{h c_{L}} K^{2} \\
-0.38 \times 10^{-7} J^{2}(J+1)^{2} \\
+0.832 \times 10^{-5} K^{2} J(J+1) \\
-0.528 \times 10^{-6} J(J+1) \\
-10.93 \times 10^{-4} K^{4}+0.264 \times 10^{-5} K^{2}
\end{aligned}
$$

for $\mathrm{ICH}_{3}$.

Applying the selection rules which are $\Delta J=1$,
$\Delta K=0$ we obtain the following rotational frequencies.

$$
\begin{aligned}
\nu_{J}^{J-1}=\frac{\hbar^{2}}{A_{0} h c_{L}} J- & 6.46 \times 10^{-6} J \\
& -1.736 \times 10^{-6} J^{3}+2.90 \times 10^{-5} K^{2} J
\end{aligned}
$$

for $\mathrm{FCH}_{3}$,

$$
\begin{aligned}
\nu_{J}{ }^{J-1}=\frac{\hbar^{2}}{A_{0} h c_{L}} J- & 3.42 \times 10^{-6} J \\
& -4.56 \times 10^{-7} J^{3}+1.952 \times 10^{-5} K^{2} J
\end{aligned}
$$

for $\mathrm{ClCH}_{3}$,

$$
\begin{aligned}
\nu_{J}{ }^{J-1}=\frac{\hbar^{2}}{A_{0} h c_{L}} J- & 1.380 \times 10^{-6} J \\
& -1.20 \times 10^{-7} J^{3}+1.340 \times 10^{-5} K^{2} J
\end{aligned}
$$

for $\mathrm{BrCH}_{3}$, and

$$
\begin{aligned}
\nu_{J}{ }^{J-1}=\frac{\hbar^{2}}{A_{0} h c_{L}} J- & 1.056 \times 10^{-6} J \\
& -1.52 \times 10^{-7} J^{3}+1.664 \times 10^{-5} K^{2} J
\end{aligned}
$$

for $\mathrm{ICH}_{3}$.

Unfortunately the pure rotation spectra of these molecules have not as yet been mapped so that comparison with experimental values must be left to the future. 